\title{
A Hybrid Selection Approach Based on Advanced Antenna Technologies for Cellular LTE-A
}

\author{
Mina Malekzadeh \\ Electrical and Computer Engineering Faculty, Hakim Sabzevari University, Sabzevar, Iran \\ E-mail: m.malekzadeh@hsu.ac.ir
}

Received: 02 January 2021; Accepted: 26 March 2021; Published: 08 April 2021

\begin{abstract}
While addressing cellular network performance issues, resolving problems with coverage and capacity is critical. The coexistence of cellular networks and smaller femtocells working within the macrocell area is among the alternative solutions. The simultaneous functioning of macrocells and femtocells, however, introduces new technical challenges that pose serious concerns about the efficiency of cellular networks. Therefore, this work proposes a hybrid approach based on variant advanced antenna technologies including MIMO methods and antenna systems for the deployment of femtocells and macrocells. To accomplish this, three distinct LTE-A network models with and without femtocells are set up with different architectures for high-density areas. The models further cover tri-sector and omnidirectional antenna systems to analyze the relevant effects on the performance of the LTE-A macrocells as well as femtocells. Also, to extend the analysis, integration of different MIMO methods for the models is provided. The networks are implemented and the link performance evaluation is carried out with regard to spectral and energy efficiency, cell and user throughputs, fairness, and SINR. The results contribute to determining the performance gains and energy saving of the LTE-A femtocells as well as macrocells by employing different advanced antenna technologies.
\end{abstract}

Index Terms: Femtocell, Macrocell, LTE-A.

\section{Introduction}

The coverage and capacity limitations are among the important challenges that cellular networks continue to face due to the rising demands for higher data rates [1]. In order to meet the cellular network requirements, it is therefore essential to address the challenges and provide high data rate communication and real-time access, particularly in dense areas. To accomplish this, among alternative solutions, this work proposes a hybrid approach for LTE advance (LTE-A) based on the implementation of femtocells and macrocells using advanced antenna technologies as multiple-input multiple-output (MIMO) methods and antenna systems.

A single-tier cellular network includes a high-power macrocell that provides coverage to a large area for the users in the cell site. However, due to constraints in the capacity, the growing number of mobile devices in dense areas will overload the macrocells and decrease the overall efficiency of the network. To avoid the issue and increase link capacity, two-tier macro-femto cellular networks are implemented. A femtocell with an omnidirectional antenna is a relatively low-power base station (less than 0.1 Watt) with small range coverage deployed in a macrocell area [2]. The deployment of femtocells expands the cellular functional range and capacity which brings certain advantages including, clear cellular signal, consistent and reliable cellular connectivity, higher spectral efficiency per coverage area, offloading traffic from the overloaded network, and higher data rate for the related users in the cellular network [1,3,4]. However, the concurrent operation of macrocell and femtocells introduces new technical challenges among which interference and unfairness in radio resource sharing are the major concerns $[5,6]$. The interference can occur due to sharing the same channel with macrocell whereas the interference with other femtocells also takes place. The interference raises serious concerns because it affects the signal's quality and results in performance degradation of the cellular network. To overcome these challenges and achieve the best performance from the implementation of femtocells, their combination with other technologies can be an alternative option [7]. In this work, the focus is on femtocell and macrocell antenna systems in combination with MIMO methods.

The MIMO methods were developed to enhance the performance of data transmission by increasing the number of radio links between the transmitter and receiver using multiple antennas. This is in contrast to the traditional single antenna port (SISO) which has one antenna on both ends of the link. There are many types of MIMO to enable a variety 
of radio links to carry the data. The transmission diversity (TxD), open-loop spatial multiplexing (OLSM), closed-loop spatial multiplexing (CLSM), multiuser MIMO (MU-MIMO) are among the most common types. The TxD method increases the signal-to-interference-plus-noise ratio (SINR) by the transmission of multiple copies of the same data and thereby, increasing the robustness of the signal to poor channel conditions and, at the same time, extending the coverage. However, despite the higher reliability, the TxD does not improve the data rate due to sending duplicate data. The spatial multiplexing methods (OLSM and CLSM), on the other hand, provides a higher data rate due to sending different data over different antennas. With regard to the MU-MIMO method, it allows the cell to communicate with multiple users simultaneously which decreases their waiting time and increases the overall network performance. However, despite the advantages, there are certain challenges to employ MIMO methods in cellular networks. Increasing the number of antennas while increases the size of the device, their improper deployment results in signal interferences because the antennas are placed in the device close to each other. In addition, the computation complexity and power consumption are higher compared to the single antenna as each antenna requires individual radio units for signal processing. Thus, an in-depth analysis is required to verify the functionally as well as the limitations of each antenna technique to adapt the systems accordingly in LTE-A cellular network.

Taking into account the growing demands for higher data rates, the main contribution of this work is to propose a hybrid approach based on the deployment of LTE-A femtocells and macrocells using variant combinations of the antenna systems and MIMO methods. The goal is to determine the most efficient combination for the LTE-A cellular network and thereby improve the performance of the end uses. The rest of this work is structured as follows. In Section 2 , the related works are discussed. Section 3 presents the details regarding the implementation and evaluation of the proposed approach. Section 4 illustrates the results and their analysis and finally, Section 5 concludes the work.

\section{Related Works}

Different studies have analyzed the challenging issues involved in providing the coverage, capacity, and data rate requirements of cellular networks. They differ in the approaches they use to alleviate the challenges. In order to improve the indoor coverage and capacity, the authors in [4] suggest a planning approach to decide the optimal location of LTE femtocells. Based on the findings, the efficiency of the network increases when exchanging traffic for position plans with a greater low superiority ratio relative to when full coverage or link quality is taken into account. The major drawbacks are that the antenna systems and MIMO methods are not taken into consideration. The authors in [5] consider the multi-tier networks with multiple antennas to addresses the massive connectivity demands of ultra-dense cellular networks. Output analyses are given in terms of coverage probability and rate coverage. Still, it is not possible to further investigate the changes in network performance using MIMO methods and other antenna systems as they are not included in the analysis methodology.

Based on the CSLM MIMO method, two different LTE networks to represent macrocell and femtocells are developed in [8]. To compare the two networks, the results in terms of throughput, average area throughput, spectral efficiency, and fairness are measured. This raises uncertainty regarding the efficiency of other MIMO methods compared to the CLSM and also omnidirectional antenna systems for which the experiments are not conducted. The round-robin, best CQI, prop fair sun, and max throughput scheduling algorithms are investigated in [9]. To determine the related impact on performance improvement of LTE femtocells, the SINR and throughput are measured. However, there is still considerable ambiguity with regard to the MIMO methods, other evaluation parameters, and antenna systems as they are ignored to address. The scheduling algorithms in LTE femtocells are also analyzed in [10] to determine how they contribute to higher data rates. The throughput, SINR, and fairness with and without mobility of the users are measured with no reference to the omnidirectional antenna systems and MIMO methods.

Beamforming and interference reduction using the coordinated multipoint transmission (CoMP) and coordinated beamforming $(\mathrm{CBF})$ are studied in order to improve data rate and capacity in femtocells [11]. The assessment is made in terms of UE selection probability and throughput. However, the downside is that no experiment and evaluation is given for the MIMO methods and antenna systems. The interference mitigation in femtocells is also taken into account to improve the network capacity for the quality of service of video data [12]. The experiments are performed to evaluate the performance in terms of throughput, packet loss rate, and mean opinion score (MOS). The disadvantage of the experiments is that they do not analyze the MIMO systems and other relevant parameters. Using an interference mitigation scheme on the basis of frequency and power domain, the efficiency and SINR improvement in femtocells are evaluated [13]. The main drawback of the scheme, though, is that it overlooks the MIMO methods, antenna systems, and other important evaluation parameters. Energy performance and throughput obtained by the deployment of femtocells for the two-tier cellular networks are investigated in [14] and [15], respectively. Nonetheless, further experiments and data analysis is required to evaluate the MIMO methods and other related significant parameters.

Despite the related works, it has not yet been identified to what extend the hybrid combination of antenna systems in macrocells as well as femtocells integrated with different MIMO methods, will satisfy the increasing demands of the LTE-A cellular network. Moreover, the selection remains unclear as to whether it is computationally demanding or energy efficient. Therefore, this work aims to expand the analysis of antenna systems as well as MIMO methods in cellular LTE-A macrocells and femtocells to establish the efficiency of the hybrid selection approach with respect to the 
requirements of cellular networks.

\section{Network Design and Implementation Methodology}

This work proposes a hybrid approach based on advanced antenna technologies including the MIMO methods and antenna systems for deployment of LTE-A femtocells and macrocells. The aim is to address the coverage and capacity needs of cellular networks and provide a vast number of users with a satisfaction data rate. To accomplish this, three different LTE-A network models are developed and implemented using MATLAB system-level simulator. The three developed network models can be seen in Fig. 1.
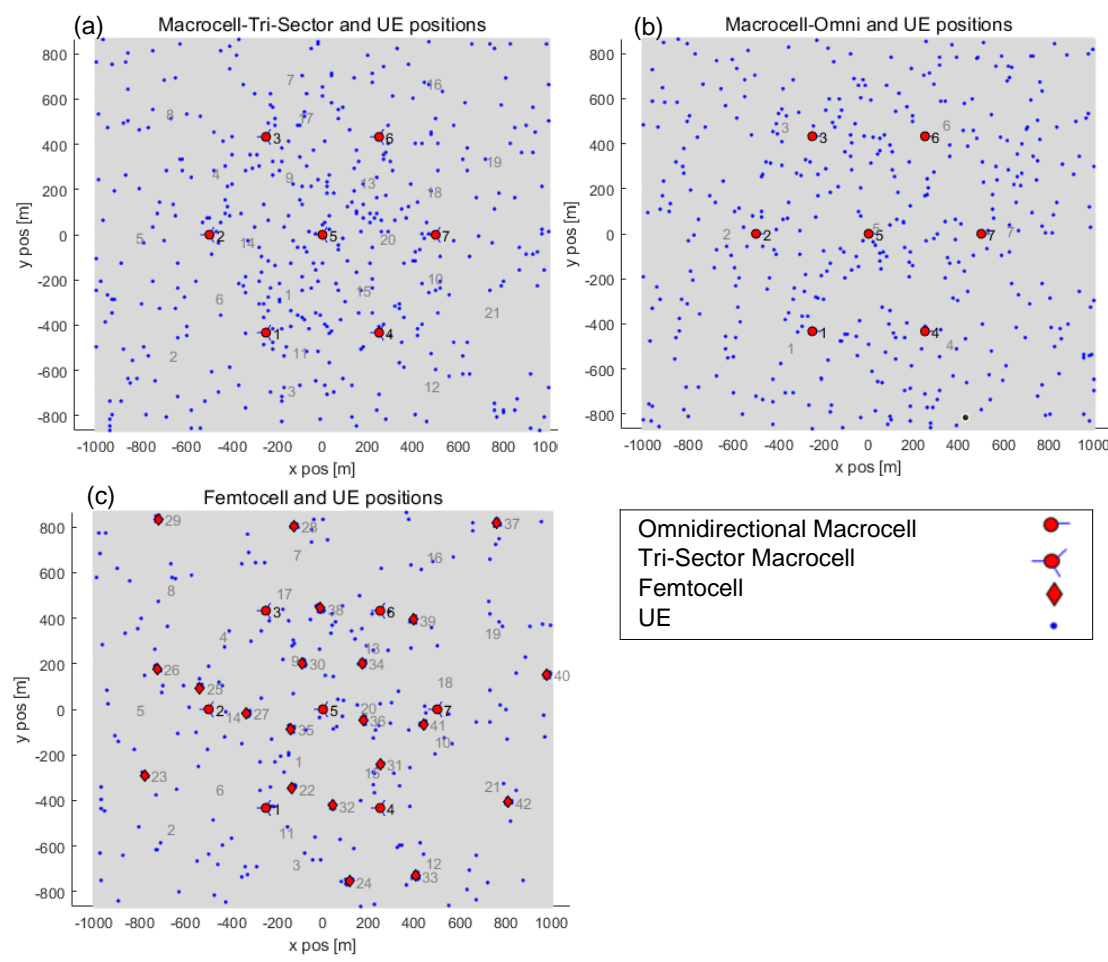

Fig. 1. Distribution of eNodeBs and UEs in LTE-A a) macrocell with tri-sector antennas, b) macrocell with omnidirectional antennas, c) macrocell with femtocells.

In all three network models, the hexagonal cell site is composed of seven macrocells with one macrocell in the central grid. Moreover, the total number of UEs is 420 in all three network models to represent a high-density area. In the first model (a), each of the seven macrocells (denoted as a red circle with three blue lines in Fig. 1 (a)) is divided into three sectors, each of which occupies a region of $120^{\circ}$ at the cell site. This provides a total of 21 cells, and given that the total number of UEs is 420 in the network, an equal number of 20 UEs are allocated to every cell with random distribution. The channel characterization for the macrocells in terms of the carrier frequency, transmission power, and channel bandwidth is defined as $2110.1 \mathrm{MHz}, 40 \mathrm{~W}$ per sector, and $20 \mathrm{MHz}$, respectively. In contrast, in the second model (b) omnidirectional antennas (denoted as a red circle with a single blue line in Fig. 1 (b)) are deployed for the macrocells in the cell site. Accordingly, in this model, each macrocell offers a coverage area for 60 UEs to distribute services equally to all 420 users. For comparison purposes, the other network parameters are defined similarly to the first model in terms of the carrier frequency, transmission power, and channel bandwidth. The third model (c), deploys several femtocells (denoted as a red diamond in Fig. 1 (c)) under the coverage of the macrocells [16]. Each one of the seven macrocells consists of three femtocells, and as each macrocell is tri-sector and is further divided into three sectors, there will be a total of 21 femtocells. Given 21 femtocells and 21 macrocells, a total of 420 users are distributed so that all the cells and femtocells equally have 10 UEs. This way, the cells cover 210 macro users and femtocells cover 210 femto users. To cover all the 420 UEs, the macrocells communicate with their attached macro UEs while each femtocell communicates with its corresponding femto UEs. All the 21 cells transmit at the same frequency as $40 \mathrm{~W}$ and all 21 femtocells also transmit at the same frequency as $0.1 \mathrm{~W}$. The other network parameters are defined in the same way as before in terms of the carrier frequency and channel bandwidth.

In addition, after the implementation of the femtocells, macrocells, and antenna systems, five different MIMO methods are integrated into each network model separately [17]. The first method is a single antenna port that does not include any MIMO method and instead uses a single antenna (SISO). The second method is based on the transmission diversity (TxD) to send identical copies of the data to multiple uncorrelated antennas. The third and fourth methods are 
based on spatial multiplexing to send independent copies of the data to multiple uncorrelated antennas as either openloop spatial multiplexing (OLSM) and closed-loop spatial multiplexing (CLSM). The fifth method, called multiuser MIMO (MU-MIMO), transmits data to multiple users simultaneously. Given these, the three network models assist in a detailed analysis for performance comparison between the macrocells and femtocells in LTE-A cellular network when the cell sites are installed with different antenna systems and MIMO methods. A description of the essential network parameters used in the network models can be found in Table 1 while the rest of the parameters are selected based on the real-word defaults [18].

Table 1. Type Sizes for Camera-Ready Papers

\begin{tabular}{l|l}
\hline Parameter & Value \\
\hline Carrier frequency & $2110.1 \mathrm{MHz}$ \\
\hline Bandwidth & $20 \mathrm{MHz}$ \\
\hline Number of cells & $\begin{array}{l}\text { First \& Third models: 21 (tri-sector) } \\
\text { Second model: 7 (omni) }\end{array}$ \\
\hline Number of femtocells & 21 \\
\hline Number of UEs & 420 \\
\hline Macro TX power & $40 \mathrm{~W}$ \\
\hline Femto TX power & $0.1 \mathrm{~W}$ \\
\hline & $\begin{array}{l}\text { Single antenna port } \\
\text { Transmission Diversity (TxD) } \\
\text { Open Loop Spatial Multiplexing (OLSM) } \\
\text { Closed Loop Spatial Multiplexing (CLSM) } \\
\text { Multiuser MIMO (MU-MIMO) }\end{array}$ \\
\hline Scheduler & $\begin{array}{l}\text { Round Robin MU for MU-MIMO } \\
\text { Round Robin for other MIMO methods }\end{array}$ \\
\hline Packet traffic model & Equal buffer model \\
\hline Path loss model & TS36942 \\
\hline
\end{tabular}

In order to evaluate the models, the performance measurements are provided with regard to spectral and energy efficiency, cell and user throughputs, fairness, and ECDF throughput and SINR parameters. The evaluated parameters are chosen on the basis of the capacity, coverage, and data rate requirements of cellular networks. The spectral efficiency is defined as the average of transmitted data rate per given bandwidth and is measured in bits per channel use (bpcu). It is an important performance parameter for data rate because it reflects the optimum utilization of the spectrum in which network transmission occurs [2]. In order to fulfill the resource constraints of the cellular devices on the user side, it is important to focus on the energy usage of these networks. The goal is to optimize the amount of data transmitted for a given amount of energy. Therefore, for link performance evaluation of the three networks, the energy efficiency [2] as the energy per bit to determine the amount of energy consumed for transmission of a single bit is measured in Joule per bit (Jpb). Moreover, the SINR of the given signal [19] and the throughput of each individual UE are also calculated as total values as well as empirical cumulative distribution function (ECDF). In addition to the total and ECDF measurements, the average throughputs of the UEs and cells are also calculated. To further determine whether the UEs are receiving a fair amount of system resources in the three network models, Jain's fairness index [2] is also measured. It is a number between zero and one so that the closer the value to one, the fairer the resources are assigned to the users of the network.

\section{Results and Discussion}

This section presents the implementation results of the three network models along with the correspondence analysis. The results are used to evaluate the approach and to determine the level of contribution of the models to performance optimization of LTE-A cellular networks with different architectures.

\subsection{SISO}

In an effort to evaluate the efficiency of the cellular LTE-A networks when the MIMO is not used for data transmissions to eliminate subsequent complexity and interference, the antenna selection here is based on a single port. Accordingly, a single antenna is integrated into all three network models and their comparison in terms of total and ECDF throughputs of the UEs are provided in Fig. 2. 

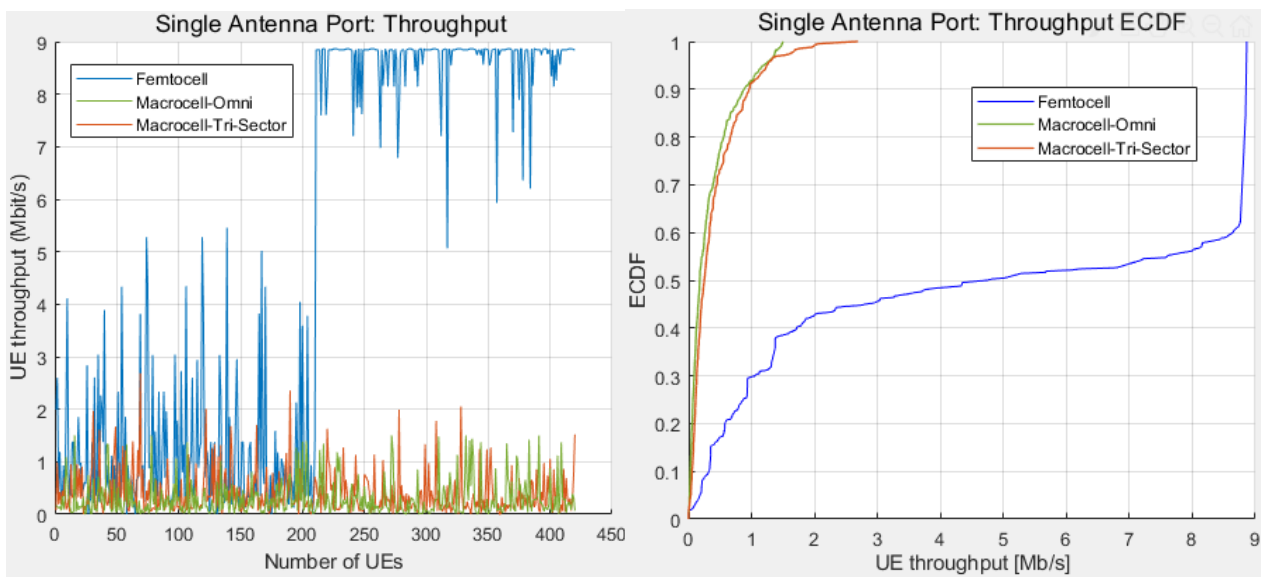

Fig. 2. Single antenna port: throughput (left) and throughput ECDF (right).

The above findings show that the LTE-A with femtocells performs much better in terms of higher data rate relative to when no femtocell is installed. Moreover, it can be found that while the femtocells significantly improve the performance of the femto users, their implementation also improves the macro users as well which is due to offloading the traffic from the macrocells. As explained in the femtocells distribution model, the approach for the femtocell network model distributes the total of 420 users in such a way that 210 users are associated with the 21 femtocells (ID: 22 to 42) and 210 users with the macrocells (ID: 1 to 21). This is why the results indicate lower throughput for the UEs (ID: 1 to 210 in the plot) that are in the coverage of macrocells compared to better throughput obtained by the UEs (ID: 211 to 420 in the plot) that are getting services from the femtocells. In this case, the average throughput is $1.17 \mathrm{Mbps}$ compared to the $8.51 \mathrm{Mbps}$ achieved by the macro and femto users, respectively. In contrast to the femtocells, a significant throughput difference is not observed when tri-sector antennas are implemented in LTE-A macrocell compared to the macrocells with an omnidirectional antenna. In this case, although the UEs in the tri-sector model achieve a slightly better data rate, the difference with the users in the omnicells is not considerable. In addition to the above throughput results, it is also important to determine the efficiency of the approach with regard to the quality and consistency of the signals received by the users. Accordingly, in terms of the SINR performance, a comparison between the three network models is given in Fig. 3.
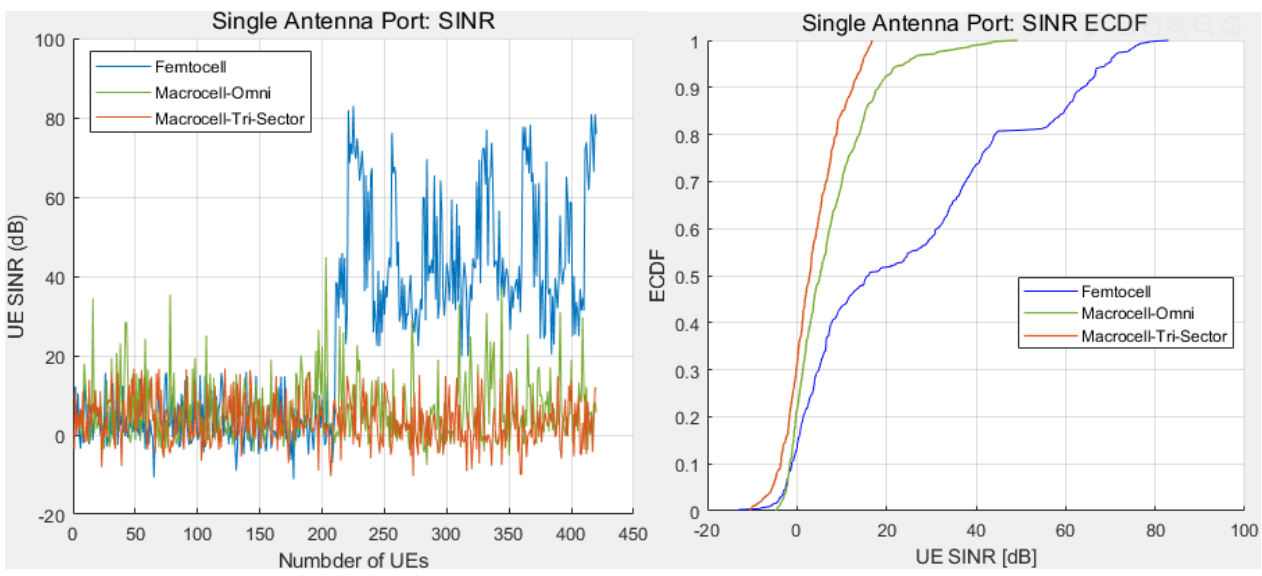

Fig. 3.Single antenna port: SINR (left) and SINR ECDF (right).

The findings of the SINR clearly indicate a comparable signal quality for the users in the macrocells without femtocell implementation as either omni or tri-sector. In contrast, the implementation of the femtocells significantly improves the signal quality. However, we find a disparity in this respect with the throughput results. Although both femto users and macro users in the network have higher data rates relative to the users in the omni and tri-sector networks, only femto users attain a higher SINR and the macro users achieve similar SINR as omni and tri-sector networks. Moreover, considering that SINR values larger than $20 \mathrm{~dB}$ indicate an excellent signal and the values between 13 and $20 \mathrm{~dB}$ indicate a good signal, the LTE-A users receive excellent signals in the second and third network models while the users receive good signals in the first model. This is important as the SINR directly affects the performance such that a higher value indicates that the signal strength is higher than the noise level and, correspondingly, increasing the data rates and network speed. Therefore, the findings give the clear idea that the femto users in the third network model receive the highest data rate as well as the strongest signals relative to the users in the coverage area of the first (tri-sector) and second (omni) network models, as well as the macro users in the third network model. 


\section{2. $T x D$}

Along with increased reliability and efficiency in MIMO methods, there are problems of computation complexity and power consumption. It is, therefore, necessary to accurately determine the performance of the MIMO communications in all three network models and adapt the system accordingly. Here, we analyze the performance of the approach when the transmission diversity method is integrated into three cellular network models. The goal is to measure the impacts and thereby determine the most efficient LTE-A network for delivering services to the corresponding UEs in the coverage area. Fig. 4 describes the contribution of each network model to throughput achievements by the UEs.
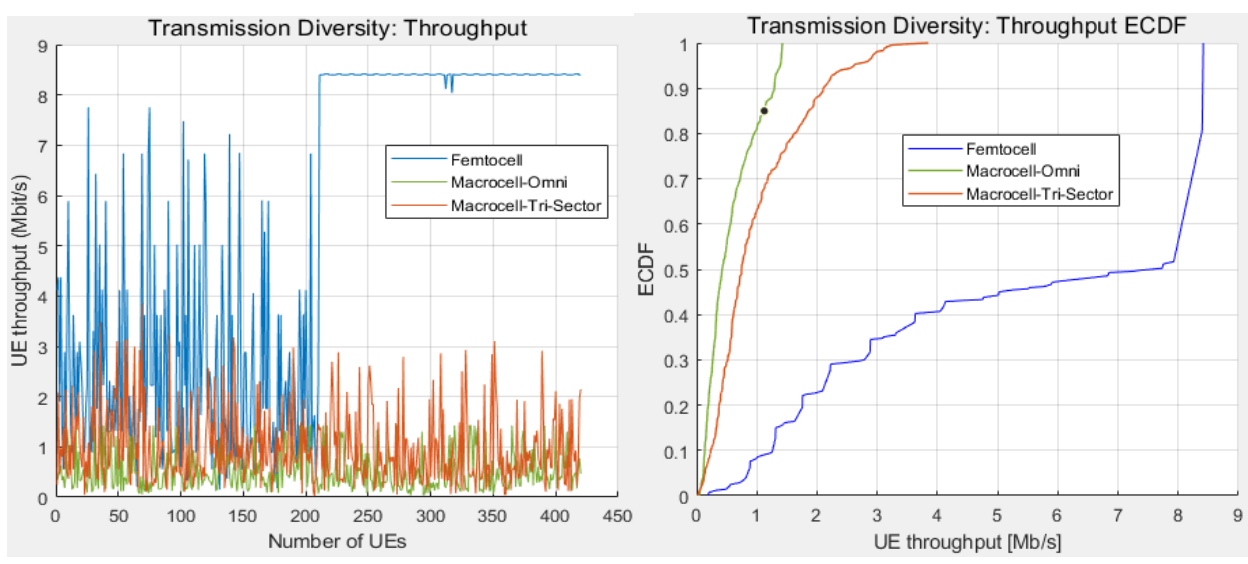

Fig. 4. Transmission diversity: throughput (left) and throughput ECDF (right).

The above findings reveal that the implementation of femtocells not only provides a higher data rate for the femto users (ID: 211 to 420) but also increases the data rate of the macro users (ID:1 to 210). This is due to the offloading traffic from the macrocells and creating less congestion in the cell sites, hence, increasing the overall data rate of the third network relative to the other two network models. In this case, the average throughput is $2.63 \mathrm{Mbps}$ compared to the $8.37 \mathrm{Mbps}$ achieved by the macro and femto users, respectively. Further on this, the users in the tri-sector cells achieve higher data rates relative to the users in the coverage area of the omnicells. A further data rate comparison between the single antenna and TxD methods implies two findings. First, considering that the reliability is defined as the ratio of successfully delivered data within the required time constraint and the total sent data, all the three network models achieve higher reliability and increased flexibility for traffic fluctuations when the TxD method is used compared to utilizing the single antenna method. This is due to mitigating the effect of multipath fading in cellular connectivity links by transferring the same data over multiple uncorrelated antennas. Second, in addition to the higher reliability, in all three network models, higher throughput values are also achieved for the LTE-A macro users using the TxD method relative to the single antenna. However, despite a much more robust data rate, the throughput using TxD is slightly lower than the single antenna for femto users in the third model, although the discrepancy is not important. On the basis of the results, the implementation of femtocells using the transmission diversity method outperforms the single antenna as well as the macrocell implementation without femtocells regardless of being omni or tri-sector. To further validate the results, the performance of the LTE-A users in the three network models is measured with regard to the SINR of the given signals. Fig. 5 shows the SINR results along with the related ECDF values.
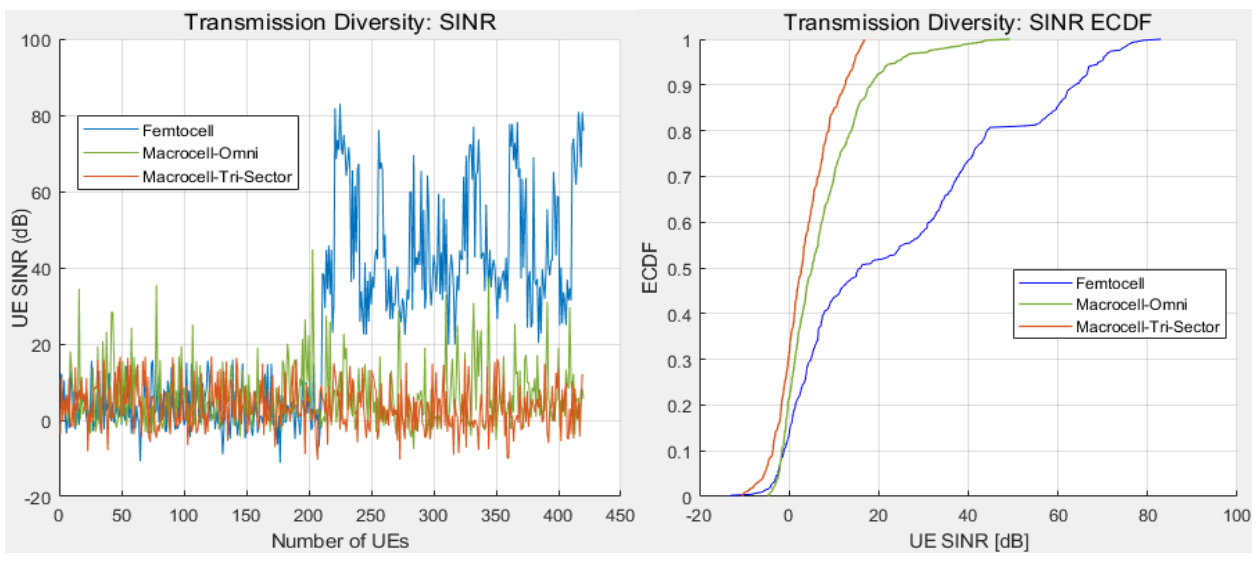

Fig. 5. Transmission diversity: SINR (left) and SINR ECDF (right). 
The above results show that the transmission diversity offers a higher signal level for the femto users in the third network model. This is in contrast to much smaller SINR values for the macro users on the same network. The users in the second model obtain higher SINR values than the first model and are relatively comparable to those of the macro users in the third model. Evidence for comparable values is given by further measurement of the SINR levels obtained by the UEs using the TxD method compared to a single antenna. This suggests that in the three network models, the use of multiple antennas for the TxD system does not have a significant benefit over the single antenna to increase the SINR strength. Therefore, on the basis of the results, we conclude that when the reliability and data rate are the main concerns, the femtocell implementation along with the TxD method will highly improve the performance compared to the single antenna method as well as omni and tri-sector implementations. In contrast, when it comes to the signal strength, no difference exists between TxD and single antenna modes while still, the implementation of the femtocells provides a higher signal strength over the omni and tri-sector.

\subsection{OLSM}

The primary purpose here is to extend the analysis with regard to adapting the OLSM MIMO method in the three network models. The comparative evaluation as a design guideline will help to further explore and understand the combined effects of the OLSM MIMO method and antenna systems as either omni or tri-sector with and without femtocells. Fig. 6 provides the results of the throughput together with the ECDF values.
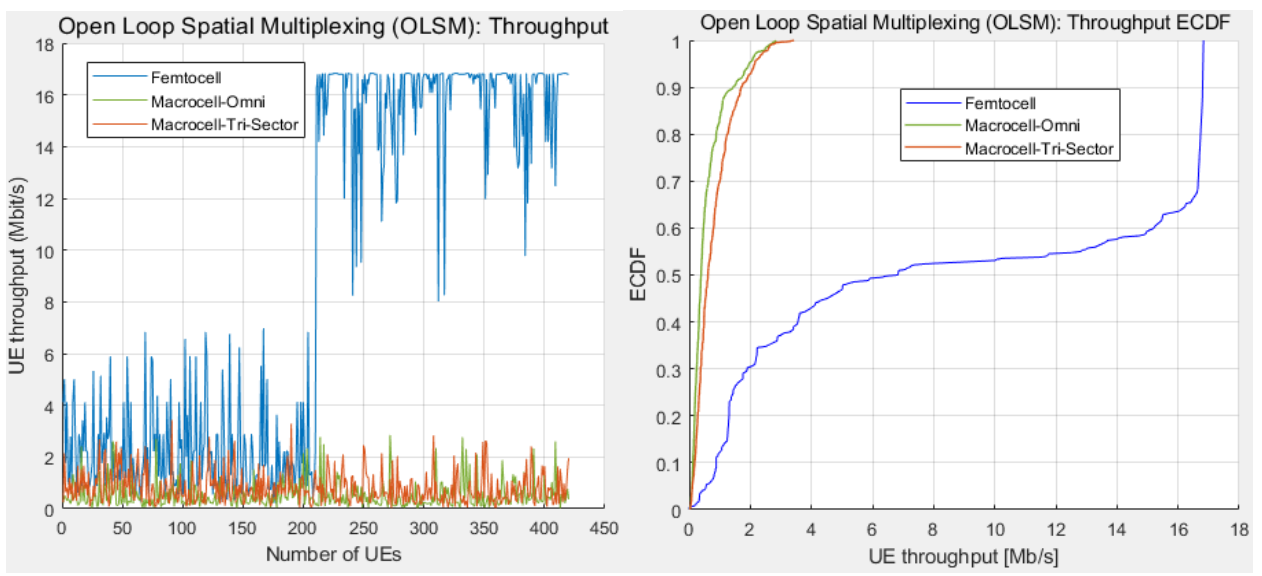

Fig. 6. OLSM: throughput (left) and throughput ECDF (right).

The above results show that employing the OLSM has a strong effect on the data rate improvement of the users when the femtocells are installed under the coverage area of the macrocells. For UEs (ID:1 to 210) that are in the coverage of macrocells, the average throughput is $2.22 \mathrm{Mbps}$ compared to the $15.57 \mathrm{Mbps}$ achieved by the UEs (ID: 211 to 420) that are getting services from the femtocells. The data rate improvement using the OLSM also includes the macro users in the third network model so that the rate is higher than the macro users in the first and second networks. The results, therefore, show no significant advantage in this regard for the implementing tri-sector cells or omnicells over each other. This means that in the case of no femtocell installation, similar data rates are provided by the first and second network models for their users. In addition, the biggest difference of the OLSM compared to single antenna and diversity methods is that the OLSM can provide a significant gain in data rate for femto users in the third network model. In this case, while the $\mathrm{TxD}$ and single antenna provide a similar data rate of about $9 \mathrm{Mbps}$ for the femto users, the OLSM provides an average of $17 \mathrm{Mbps}$ which is almost twice the amount. Concerning the omnicells, using the OLSM is a better selection than using either single port or TxD methods in terms of higher data rate. This means that the UEs in the omnicell network using the OLSM MIMO achieves higher speed than those with the single antenna or TxD methods in the same network. However, the OLSM and TxD methods provide the same level of data for the trisector users which is higher than the users with a single antenna port in the same network. This conclusion can also be verified by Fig. 7 where the relationship between data rate and SINR can be identified. 

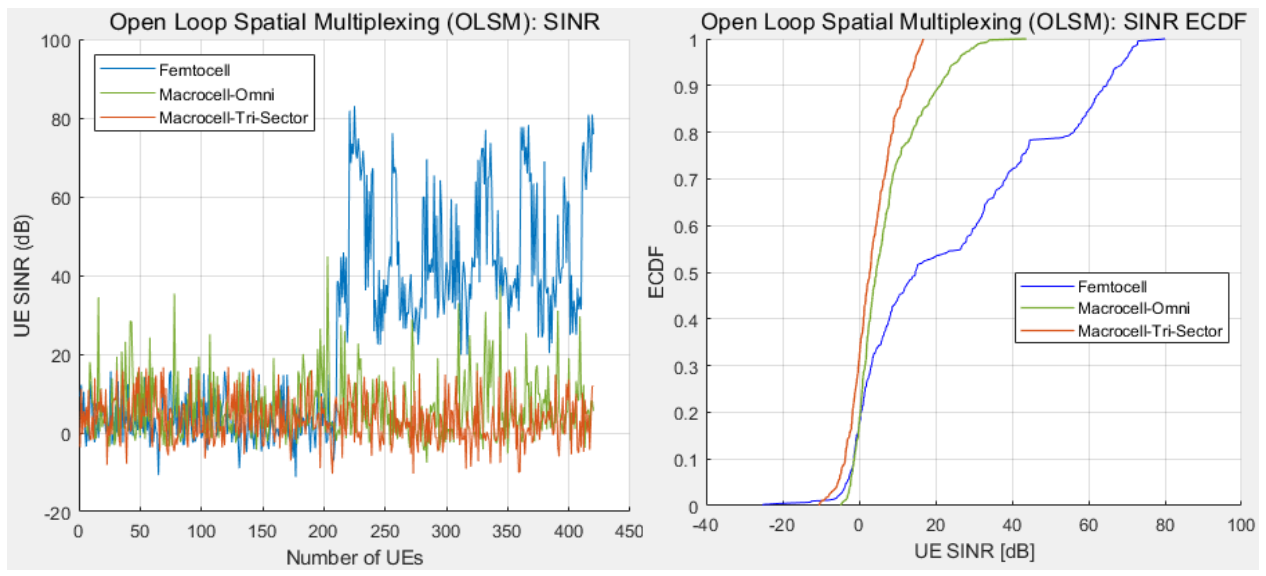

Fig. 7. OLSM: SINR (left) and SINR ECDF (right).

The findings show that the femto users achieve a much better signal level compared to the macro users in the same network as well as the macro users in the omni and tri-sector networks. In this case, although the SINR of the macro users in all three networks is lower than the femto users in the third network, they still receive SINR above $13 \mathrm{~dB}$ which is in an acceptable range. In addition, from the SINR results it can be seen that even though the OLSM has the potential to significantly enhance the throughput, its use does not change the signal level compared to the single antenna and TxD methods in the same network models. Therefore, when the UEs are served by spatial multiplexing, they achieve the same level of signals compared to the UEs that use the single antenna and TxD for their communication. This is related to the fact that spatial multiplexing is effective when the UEs have a high SINR so data will be decoded well. Among the three network models, the femto users in the third network have the highest SINR values about 80dB compared to macro users in the third model and other users in the first and second models with SINR close to 20dB. This is the reason why using the OLSM method can highly improve the data rate of the femto users in contrast to the TxD method. Therefore, when the SINR is low and no UEs can be served by spatial multiplexing, it is an energy-efficient strategy to not use this method and thereby reduce the power consumption.

\subsection{CLSM}

In this section, the data rate challenges are evaluated with regard to the effects of the CLSM MIMO on the performance of macrocells with omnidirectional and tri-sector antennas relative to the macrocells that deploy femtocells under their coverage. The effects on throughput are given in Fig. 8 along with the ECDF values.

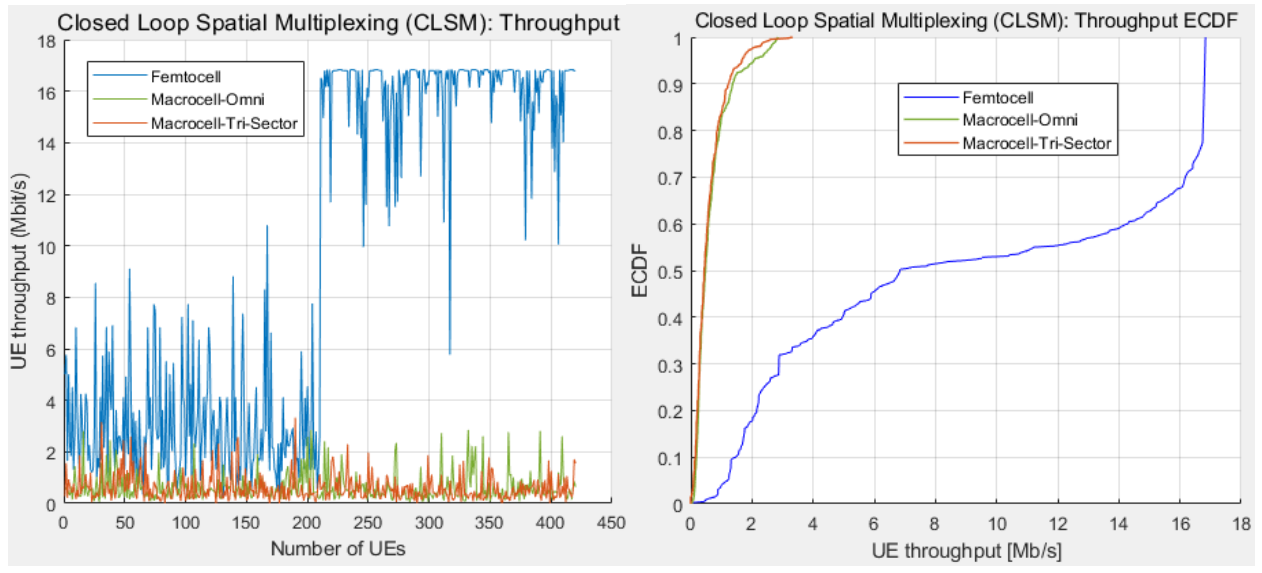

Fig. 8. CLSM: throughput (left) and throughput ECDF (right).

The results show that to achieve a reliable connection, the implementation of the femtocells is the best selection compared to the omni and tri-sector cells. The femtocell deployment using the CLSM method provides higher data speed for both macro and femto users, while the latter achieve more benefits. For UEs (ID:1 to 210) that are in the coverage of macrocells, the average throughput is $3.08 \mathrm{Mbps}$ compared to the $15.34 \mathrm{Mbps}$ achieved by the UEs (211 to 420) that are getting services from the femtocells. In contrast, when no femtocell exists, the macro UEs using the CLSM method in the first and second network models achieve similar data rates they are lower than those of the macro UEs in the third network model. Further comparison shows that choosing between open- and closed-loop MIMO methods does not bring any specific advantages over each other in the three network models. In contrast, using the CLSM method over the single antenna and TxD results in significant data rate improvement for the users of the third network with 
femtocell deployment. To extend the CLSM analysis, the strength of the receiving signals by the UEs are measured to assess the efficiency of the three network models. The SINR signal level and the related ECDF values are presented in Fig. 9.
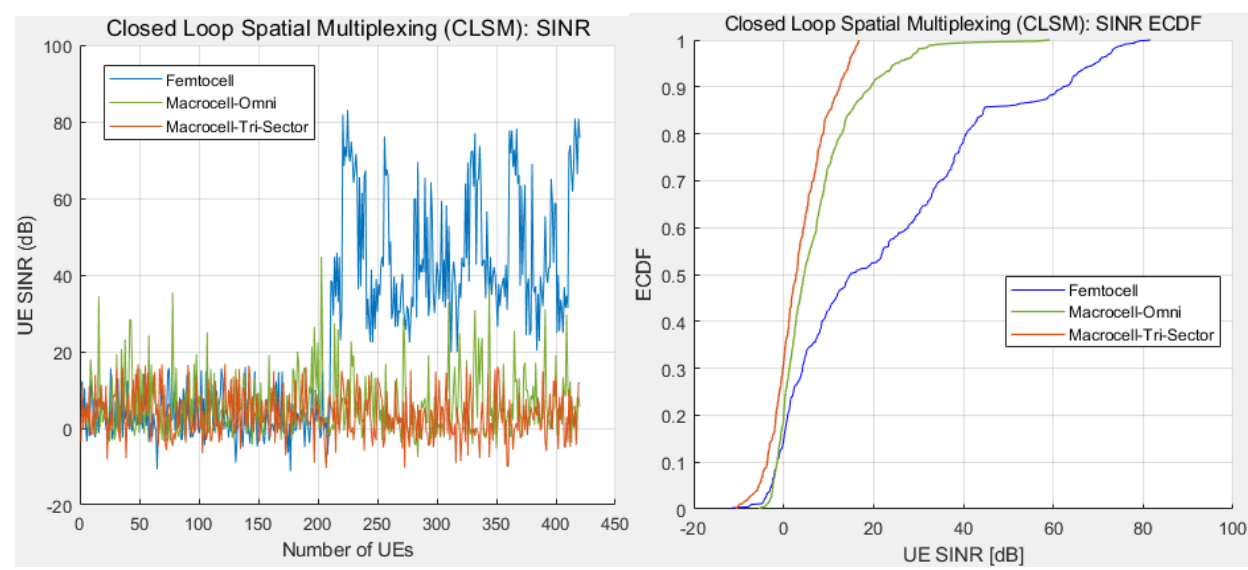

Fig. 9. CLSM: SINR (left) and SINR ECDF (right).

The SINR results clearly contrast with the throughput findings on better performance of femto users in terms of getting a much higher level of signal strength. With regard to macro users, they all achieve a similar level of SINR regardless of being in which of the three networks. Further SINR level comparison of the CLSM and single antenna, TxD, and OLSM reveals that although no major SINR changes are observed for the macro UEs in the first and third network models, the SINR in the second network can be improved using the CLSM method compared to the other methods. In this case, in the tri-sector macrocell, the SINR rises from 50dB to 60dB. It is also inferred from the results that special multiplexing methods perform much better than the transmit diversity or single antenna methods. Moreover, where the SINR is the primary concern, the use of CLSM is more advantageous than OLSM because the impact of noise becomes minor. In this regard, in order to achieve the highest performance, the deployment of femtocells with the CLSM will enhance the performance of the users compared to omnidirectional and tri-sector deployment using either single antenna, TxD, or OLSM methods.

\subsection{MU-MIMO}

In MU-MIMO systems, several UEs are scheduled at the same time to provide increased link reliability and data rate. Here, we investigate the possible advantages for LTE-A users by using spatial multiplexing to divide the data streams towards multiple UEs in all three cellular network models. The relationship between the MU-MIMO method and the antenna systems is characterized by data rate efficiency presented in Fig. 10.


Fig. 10. MU-MIMO: throughput (left) and throughput ECDF (right).

As shown in the above results, it is confirmed that the femtocell deployment can greatly enhance the data rate of the users. In this regard, the femto users reach a much better data rate than the macro users in the third network model. For UEs (ID: 1 to 210) that are in the coverage of macrocells (1 to 21), the average throughput is 3.29Mbps compared to the $13.51 \mathrm{Mbps}$ achieved by the UEs (ID: 211 to 420) that are getting services from the femtocells. The macro users' comparison in all three network models further confirm their better performance in the third network. Moreover, from the results, it can be seen that the MU-MIMO provides better performance for the macro users under the coverage of the 
tri-sector cells (second network model) than the users that are in the omnidirectional cells (first network model). Further data rate comparison between the MIMO methods shows that for tri-sector cells, the MU-MIMO results in a higher performance of the users in this network than either single antenna and close- and open-loop spatial multiplexing methods. With regard to the omnicells and femtocells, using the MU-MIMO along with the CLSM and OLSM methods provide the users with a higher data rate than the single antenna and transmission diversity. These findings as the average values have been summarized in Fig. 11.
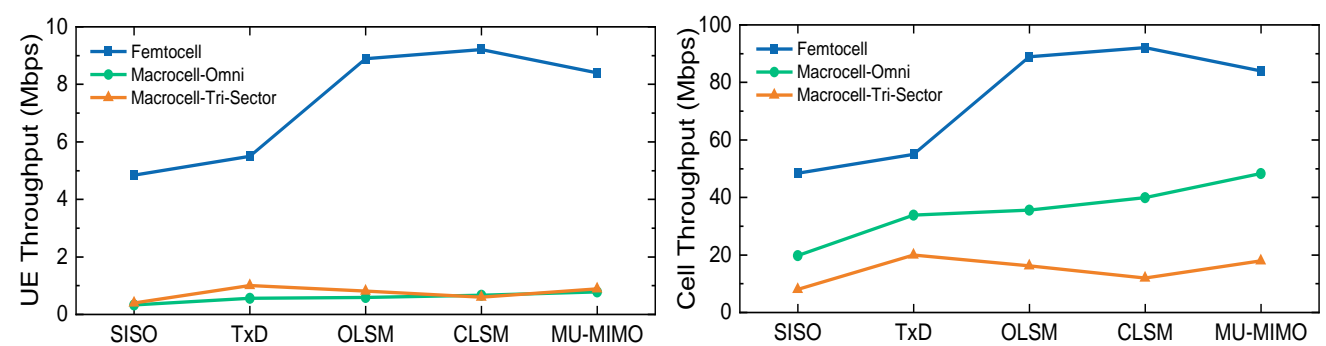

Fig. 11. Average UE throughput (left) and cell throughput (right).

To validate these findings, the SINR measurement of the MU-MIMO methods in the three network models are obtained and shown in Fig. 12.
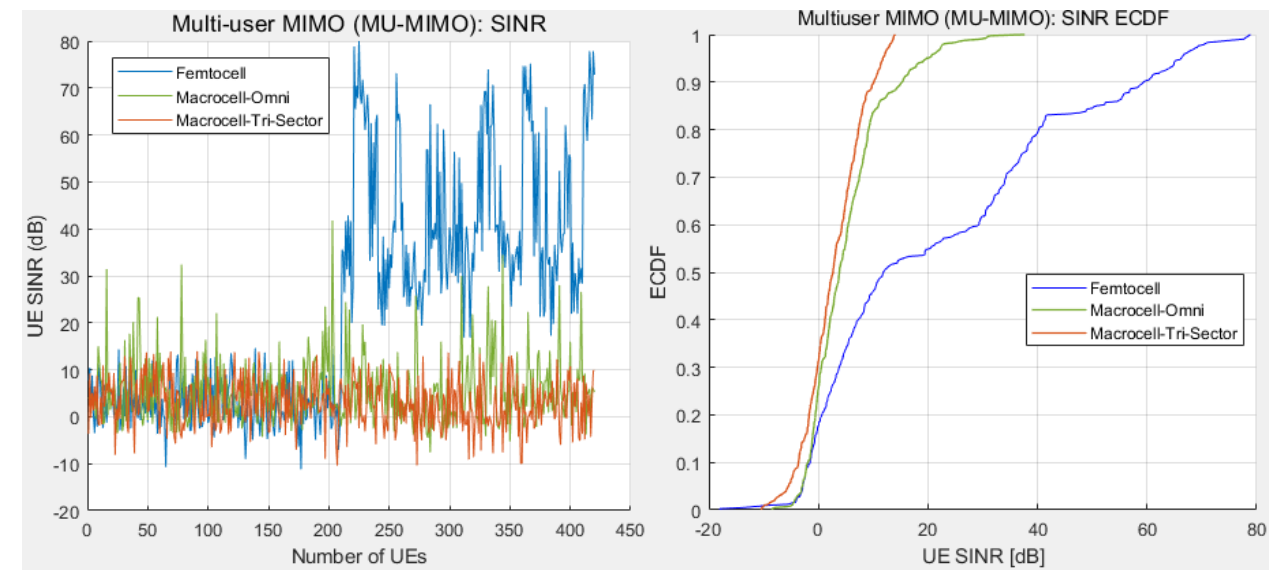

Fig. 12. MU-MIMO: SINR (left) and SINR ECDF (right).
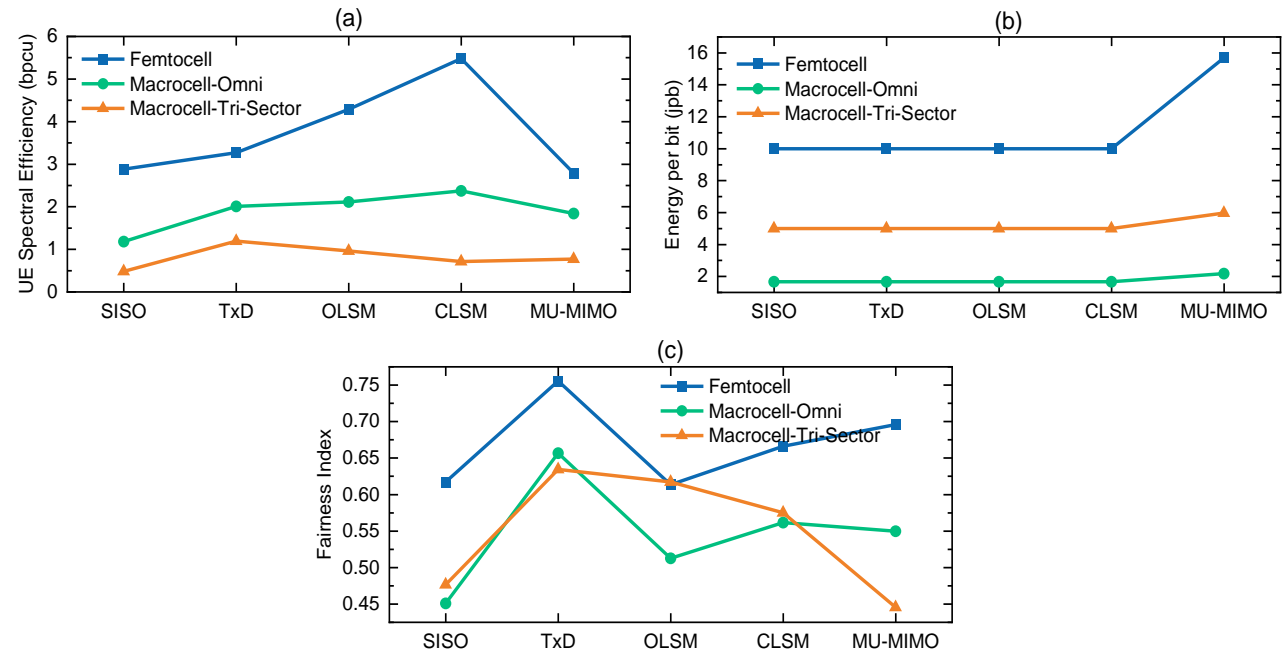

Fig. 13. The average performance comparison of the network models: a) spectral efficiency, b) energy efficiency, c) fairness index.

The results show that the users in the femtocells receive a far higher level of SINR compared to all other macro users in the three networks. The highest signal for femto users is $80 \mathrm{~dB}$, which corresponds to an excellent signal level relative to noise. The macro users in the omnicells also capable of $38 \mathrm{~dB}$ at the highest level, which is in comparison to $14 \mathrm{~dB}$ for users in the tri-sector cells. Although the SINR of the macro users in the second network is lower, the signal 
strength remains in an acceptable range. In order to further analyze the performance of the three networks, their performance is measured to assess the fairness along with the energy and spectral efficiency. The results are provided in Fig. 13.

The higher spectral efficiency of the CLSM method shows that, among the MIMO methods in LTE-A cellular communication, it is capable of allowing more effective use of the limited frequency spectrum and thereby improving the data transmission rate over the given bandwidth in femto and omnicell networks. On the other hand, in the tri-sector network, while the difference between the MIMO methods is not as significant as in the femtocells, the transmission diversity method provides a better bandwidth usage. The energy efficiency results also confirm that the femtocell deployment can better preserve the energy required for the successful transmission of the information bits. A further comparison reveals that, in order to maximize the battery lifetime, the tri-sector is a better deployment than omnidirectional as it reduces the energy consumption and extends the network lifetime. With regard to the MIMO methods, the use of MU-MIMO requires more energy for transfer of bits compared to the other four methods. The results also demonstrate that using femtocells, the LTE-A is able to provide much higher fairness to distribute the resources between the UEs relative to the tri-sector and omnicells. In this regard, the TxD can highly improve the resource allocation in contrast to the single antenna method which has the least fairness.

\section{Conclusion}

This work contributes to providing a hybrid approach for improving the performance of LTE-A cellular communications. The approach is based on advanced antenna technologies such as antenna systems, including femto, omni, and tri-sector implementations, as well as MIMO methods including TxD, CLSM, OLSM, and MU-MIMO. To accomplish this, the approach includes three network models with different architectures for implementation of which the MATLAB system-level simulator is used. The findings demonstrate the better performance of the LTE-A network with femtocell deployment compared to the omni and tri-sector networks in all of the evaluated aspects. If femtocells are deployed, using the CLSM method improves the performance compared to other methods in terms of energy and spectral efficiency, throughput, and SINR. However, the fairness of resource allocation is higher when the TxD method is used in the femtocells. On the other hands, if the cell sites do not contain femtocells, the omni and tri-sector cells demonstrate their benefits in different aspects. The omnicells outperform tri-sector cells in terms of cell throughput, SINR, and spectral efficiency when the CLSM method is used. As far as the UE throughput is concerned, there is no clear distinction between MIMO methods in tri-sector and omnicells. When it comes to energy consumption, the highest efficiency can be achieved in tri-sector cells using all the MIMO methods. The findings establish a baseline to select the optimal configurations that effectively optimize the performance of users in LTE-A while as a part of future work they can be further extended for optimization analysis of $5 \mathrm{G}$ cellular networks.

\section{References}

[1] J.K.N. Nyarko and C.A. Mbom. A Performance Study of Massive MIMO Heterogeneous Networks with Ricean/Rayleigh Fading. MDPI journal of Electronics, Vol. 7, No.6, 2018.

[2] S. Rajoria, A. Trivedi, and W.W. Godfrey. comprehensive survey: Small cell meets massive MIMO. Elsevier Physical Communication, Vol. 26, 2018.

[3] M.A. Adedoyin and O.E. Falowo. Combination of Ultra-Dense Networks and Other 5G Enabling Technologies: A Survey. IEEE Access, 2020.

[4] J.M.R. Avilés, M. Toril, and S.L. Ramírez. A femtocell location strategy for improving adaptive traffic sharing in heterogeneous LTE networks. Springer URASIP Journal on Wireless Communications and Networking, 2015.

[5] M.M. Fadoul. Modeling multi-tier heterogeneous small cell networks: rate and coverage performance. Springer Telecommunication Systems, 2020.

[6] O. Alamu, A.G. Ilori, M. Adelabu, A. Imoize, and O. Ladipo. Energy efficiency techniques in ultra-dense wireless heterogeneous networks: An overview and outlook. Elsevier Engineering Science and Technology, an International Journal, Vol. 23, 2020.

[7] S.F. Yunas, W.H. Ansari, and M. Valkama. Technoeconomical Analysis of Macrocell and Femtocell Based HetNet under Different Deployment Constraints. Hindawi Mobile Information Systems, 2016.

[8] S.F. Abied, A.B. Shams, and M.T. Kawser. Comparison of the LTE Performance Parameters in Different Environments under Close Loop Spatial Multiplexing (CLSM) Mode in Downlink LTE-A. Journal of Computer and Communications, Vol. 5, No. 9, 2017.

[9] B. Krasniqi and B. Rexha. Analysis of Macro-Fem to Cellular Performance in LTE Under Various Transmission Power and Scheduling Schemes. Journal of Communications Vol. 13, No. 3, 2018.

[10] A. Noliya1 and S. Kumar. Performance Analysis of Resource Scheduling Techniques in Homogeneous and Heterogeneous Small Cell LTE-A Networks. Springer Wireless Personal Communications, 2020.

[11] G.G. Lema. Performance evaluation of beamforming for network throughput enhancement. Wiley International journal of communication systems, 2020.

[12] T.A. Yahiya and P. Kirci. Performance Study of Adaptive Video Streaming in an Interference Scenario of Femto-Macro Cell Networks. MDPI journal of Information, Vol.9, No.1, 2018. 
[13] S. Sbit, M.B. Dadi, and B.C. Rhaimi. Interference reduction in long-term evolution-advanced by frequency and power domains. Wiley Internet Technology Letters, 2019.

[14] R. Tian, L. Ma, Z. Wang, and X. Tan. Cognitive Interference Alignment Schemes for IoT Oriented Heterogeneous Two-Tier Networks. MDPI journal of Sensors, 2018.

[15] H. Malik, J.L.R. Sarmiento, M.M. Alam, and M.A. Imran. Narrowband-Internet of Things (NB-IoT): Performance Evaluation in 5G Heterogeneous Wireless Networks. IEEE 24th International Workshop on Computer Aided Modeling and Design of Communication Links and Networks (CAMAD), Limassol, Cyprus, 2019.

[16] S. Kilaru and A. Gail. Improving Quality of Service of Femto Cell Using Optimum Location Identification. MECS International Journal of Computer Network and Information Security, Vol. 7, No. 10, 2015.

[17] S. Ravindra and G.K. Siddesh. Interference Mitigation and Mobility Management for D2D Communication in LTE-A Networks. MECS International Journal of Wireless and Microwave Technologies, Vol. 2, No. 2, 2019.

[18] 3GPP Technical Specification. LTE; Evolved Universal Terrestrial Radio Access (E-UTRA); User Equipment (UE) radio transmission and reception (3GPP TS 36.101 version 10.3.0 Release 10), 2011.

[19] S. Murugan. Compact MIMO Shorted Microstrip Antenna for 5G Applications. MECS International Journal of Wireless and Microwave Technologies, Vol. 11, No. 1, 2021.

\section{Author's Profile}

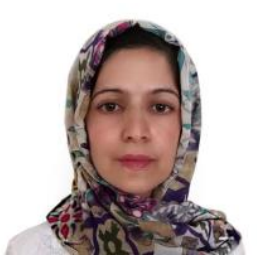

Mina Malekzadeh received the M.Sc. degree in software engineering and the Ph.D. degree in computer security from Universiti Putra Malaysia (UPM University) in 2007 and 2011, respectively. She is currently an Assistant Professor with the Department of Computer Engineering, Hakim Sabzevari University. Her main research interests include computer networks, wireless and mobile communication systems, network security, IoT, and big data.

How to cite this paper: Mina Malekzadeh, " A Hybrid Selection Approach Based on Advanced Antenna Technologies for Cellular LTE-A", International Journal of Wireless and Microwave Technologies(IJWMT), Vol.11, No.2, pp. 1-12, 2021.DOI: 10.5815/ijwmt.2021.02.01 